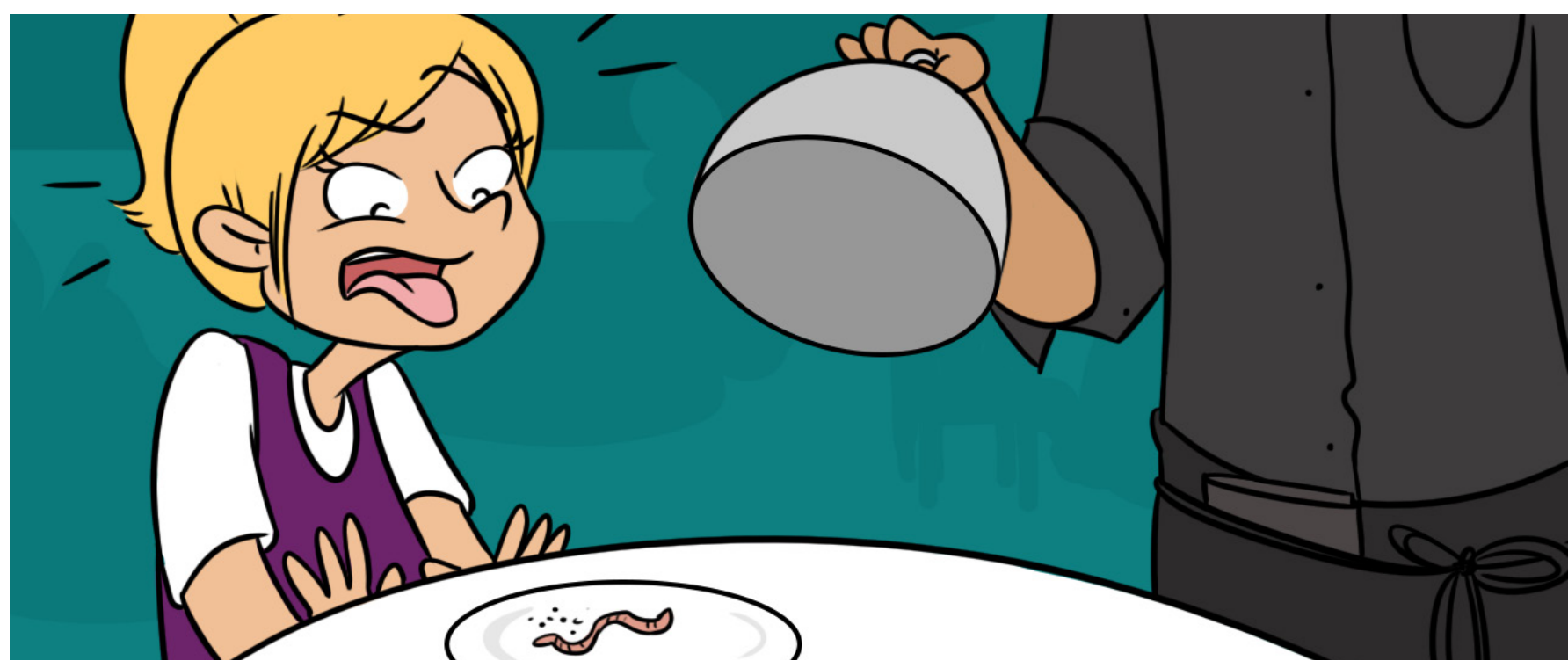

\title{
EATING WORMS TO TREAT AUTOIMMUNE DISEASES?
}

\section{Nerissa Ora Zyskind Tunnessen ${ }^{1}$ and Michael H. Hsieh ${ }^{1,2,3 *}$}

'Biomedical Research Institute, Rockville, MD, United States, '2Division of Urology, Children's National Health System, Washington, DC, United States, ${ }^{3}$ Department of Urology, George Washington University, Washington, DC, United States

\section{REVIEWED BY:}

ANNIE AGE: 14
Can eating worms actually make you healthy? Most people do not think of parasitic worms and disease treatments as going together. Recently, researchers have found that a certain kind of parasitic worms, called helminths, may have health benefits, specifically in autoimmune diseases. Autoimmune diseases are diseases in which the body attacks healthy cells or recognizes positive "self" cells as dangerous. The idea of using helminths for autoimmune disease treatment goes back to 1989, when a scientist named David Strachan proposed something called the hygiene hypothesis, which is the idea that the immune system is some what dependent on the environment. Helminth treatment is also connected to the hypothesis that exposure to bacteria and other microbes can help to build up the immune system. It is possible that helminths help to regulate the functions of the body, particularly in people who are more likely to have an autoimmune disease. So far, researchers have found that helminths - and molecules from helminths - decrease inflammation and symptoms of autoimmune diseases in animal experiments and in some humans. 
HELMINTHS

Parasitic worms.

AUTOIMMUNE DISEASE

A disease in which the immune system attacks the body's healthy cells.

HYGIENE HYPOTHESIS

A hypothesis that states that a lack of exposure to infectious agents increases a person's likelihood of getting allergic and autoimmune diseases.

\section{WHAT IS A HELMINTH?}

Helminths are parasitic worms that affect more than 1.5 billion people around the world. These worms can live in many different organs and can cause serious harm to the body. Many helminths are organ-specific, like Schistosoma haematobium, which lives in the veins of the human bladder. Although the symptoms of infection with these the worms differ depending on the type of helminth, many infections do not have immediately noticeable symptoms, being asymptomatic, but may lead to dangerous long-term problems. Thus, a person can often live for years without realizing that they have a helminth infection, but could later suffer from like cancer or organ failure.

One example of a helminth infection is schistosomiasis, which comes from an infection with a type of helminth called a schistosome. Schistosomes can lead to an enlarged liver, liver failure, anemia, bladder cancer, and many other dangerous long-term effects. Some species of helminths, however, cause infections that are very easy to see. For example, in certain cases of infections caused by larva of some worms, something called cutaneous larva migrans, or "creeping eruption," can occur, which can be visible to the naked eye. It can look like poison ivy rashes or like a worm digging through the skin. This occurs most commonly on the sole of the foot, where the worms burrow into the skin and can cause intense itching.

Although these worms are common, they are treatable with medications that vary from worm to worm. It is important to understand that, although helminths can cause extremely dangerous conditions, they can be treated in most cases.

\section{THE OLD FRIENDS HYPOTHESIS}

In 1986, David Strachan proposed that lifestyle changes in industrial countries that have led to the decline of "infectious burden" are associated with the rise of allergic and autoimmune diseases. "Infectious burden" refers to infectious diseases like viruses, parasites, and pathogenic bacteria. This is known as the "hygiene hypothesis," or the idea that the immune system is somewhat dependent on environmental exposures to infections. This hypothesis was developed by looking at how the spread of infectious diseases and autoimmune and allergic diseases were connected to each other. It was observed that there were nearly no instances of allergic or autoimmune diseases in areas with high instances of some infectious diseases.

Some common infectious diseases include the hepatitis A, gastrointestinal infections, and parasitic infections (including those caused by helminths). These infectious diseases have been essentially wiped out in many developed 
OLD FRIENDS HYPOTHESIS

A hypothesis that states that since microbes (like bacteria and helminths) evolved with the immune system, they have an effect on the immune system that is needed for its proper function. countries, but these are the same areas in which the frequency of autoimmune diseases has skyrocketed. Autoimmune diseases like asthma, allergic rhinitis (seasonal allergies), atopic dermatitis (eczema), and inflammatory bowel diseases (ongoing inflammation of the digestive tract) affect a large number of people, particularly within developed, industrialized, and economically advanced countries. In these countries, autoimmune diseases affect roughly $20-40 \%$ of the population and the number of affected individuals continues to increase. This increase in the occurrence of autoimmune diseases is important to pay attention to because many of these diseases can be life-threatening.

There have been many studies investigating the relationship between the exposure to infections and the development of autoimmune diseases. Many of these studies have been done in animals, or by examining immigrant populations and their children. In families where the parents grew up in areas with lots of helminth infections, children, who grew up after their families immigrated to the areas with better hygiene seem to be more likely to develop allergic and autoimmune disorders than their parents, probably because of differences in the environment.

An extension to the hygiene hypothesis that included helminths came in 2004. This extension suggested that the huge increases in allergies, inflammatory bowel diseases, and autoimmune diseases may have to do with the absence of helminths in the environment. This is known as the old friends hypothesis [1], playing off of the idea that since helminths have infected humans for as long as we know (Otzi the iceman, who lived between 3400 and 3100 BCE had a helminth infection) and are thus "old friends." Since then, there have been many studies in both humans and mice that have shown correlations between helminth infections and these diseases. One study showed a decrease in inflammation in a model of colitis (an inflammatory bowel disease) when proteins from the helminths Schistosoma mansoni and Ascaris caninum were given [2]. Another experiment showed that larvae of the worm Litomosoides sigmodontis could slow down how fast type 1 diabetes expressed in mice. In human studies, the helminths Necator americanus and Trichuris suis were shown to significantly improve symptoms associated with Crohn's disease and ulcerative colitis (both inflammatory bowel diseases) [3].

These studies are important to the understanding of the old friends hypothesis, because they point to possible uses of helminths as new medications for potentially life-threatening autoimmune diseases.

\section{HELMINTHS HAVE ANTI-INFLAMMATORY PROPERTIES}

In autoimmune diseases, the immune system attacks the body and causes unnecessary and harmful inflammatory responses. Research into helminths 
TH2 RESPONSE

A type of immune response against helminths and other parasites that often is anti-inflammatory. It helps to directly trap, kill, and expel parasites while also stimulating the repair of damage the parasites caused.

\section{FIGURE 1}

The helminth immune response has three major parts: the macrophages, the Th2 response, and the $T$ regulatory response. Helminths spurr the activation of immune cells called macrophages which promote the repair of tissues like organs, muscles, and ligaments. Helminths also attract dendritic cells, which are antigen-presenting cells. This means that they activate a T-cell response which is more specific to certain antigens. A T-effector cell (Th0) will give rise to T-helper-2 cells (Th2) and T regulatory cells. Th2 cells give rise to mast cells and basophils, which serve as inflammatory mediators, B-cells produce immunoglobulin E (lgE) which can cause itching and rashes, and goblet cells help to increase mucus production, which can help to regulate inflammatory bowel diseases in particular. has shown that many of these parasites cause an anti-inflammatory response from immune system called the Th 2 response, or the T-helper cell 2 response, which decreases inflammation. This response helps the immune system to directly trap, kill, and expel parasites. The Th2 response also stimulates repair of the physical damage caused by the worms, which also helps the antiinflammatory response (Figure 1).

The immune response to helminths begins when a dendritic cell is attracted to the helminth. Dendritic cells are something called antigen-presenting cells, which means that they use whatever is infecting the body to shape the cell and then call another cell. More specifically, dendritic cells go to an essentially unformed cell called a Th0 cell, which is important to a specific immune response. These Th0 cells change into other types of Th cells depending on what signals the dendritic cell gives it. In a Th2 response, the Th0 cells will give rise to a Th2 cell. Th2 cells activate various other immune cells that serve as a part of the anti-inflammatory response. These cells include mast cells and basophils (which are control parts of inflammation), B-cells (which produce antibodies), and goblet cells (which increase the production of mucus, helping especially to regulate inflammatory bowel diseases).

Immune cells called macrophages that are also simulated by the Th2 response help to promote the repair of damaged tissues, such as organs, muscles, and ligaments. The Th2 response also causes the production of an antibody called

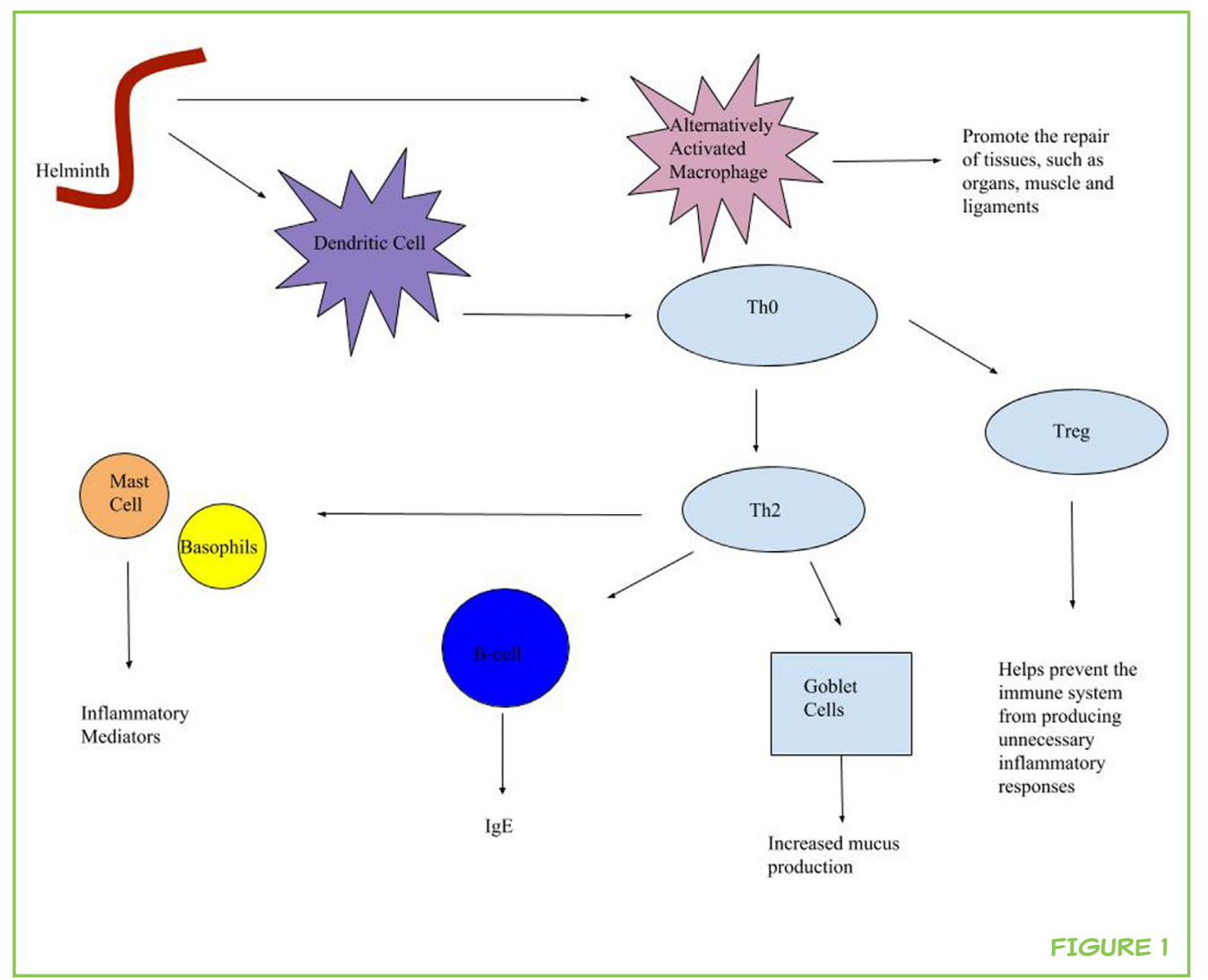


immunoglobulin E, which causes itching and rashes. Another important part of the Th2 response that helps to control the immune system are T-regulatory cells, or Tregs, which help to regulate the immune system so that it does not produce too much inflammation. Some helminths are thought to be able to activate Tregs, likely in order to protect themselves from the immune system, since Tregs tend to make it more difficult to get rid of the parasites. Tregs, however, help the person or animal to avoid over-responding to other things in the environment, meaning that they decrease allergic reactions and the symptoms of autoimmune diseases. But, they can be a disadvantage to the body as Tregs activated can lead to long-term helminth infections.

Although some worms seem to help to control the immune response to allergens by limiting inflammation, other worms can be very dangerous to preexisting conditions - for instance, if someone already had an autoimmune disease-by worsening them. So, it is important to understand which worms could be used to treat autoimmune diseases and which worms should not.

\section{NOT JUST THE WORMS: HELPFUL MEDICAL USES}

Since the discovery of the ability of helminths to regulate the immune system, some researchers have begun to search for ways to use helminths to treat autoimmune diseases. Now, "helminth therapy" has become a somewhat popular, last-resort method of treatment for severe autoimmune diseases. There are many cases of people who seem to have dramatic successes in treating their life-threatening ulcerative colitis (an irritable bowel disease) by swallowing whipworm eggs (T. suis and Trichuris trichiura).

In a 2016 New York Times article, Velasquez-Manoff reported on the practice of ingesting helminth eggs to help get rid of severe autoimmune disorders [4]. He interviewed multiple helminth users for this article. Vik, one of the people he interviewed, suffered from severe ulcerative colitis. His medications had failed, and he risked developing even more life-threatening conditions. After reading about the hygiene hypothesis and a small scale experiment performed by a scientist who used T. suis to treat inflammatory bowel diseases (with $43 \%$ of ulcerative colitis patients showing improvements after 12 weeks), Vik decided to try whipworm eggs. He traveled to Bangkok and obtained a vial of human whipworm (T. trichiura) eggs from a local parasitologist (someone who studies parasites), and then ingested the eggs several months later. Vik was able to decrease the amount of medication that he had been using to treat his ulcerative colitis, and his symptoms faded, flaring up only occasionally as the whipworms he ingested grew old and diminished. In 2011, Vik's case study was published as an inspiration for others to follow suit. 
In addition to Vik, many people have been using worms to treat their autoimmune diseases. However, this can be extremely dangerous since helminths can have life-threatening effects on the human body. As a result, some scientists have started studying the molecules secreted by the worms. In some diseases, these molecules may have beneficial because they can the immune system. Many of the molecules secreted by the worms that have been studied stimulate a Th2 response. Some molecules have shown antiinflammatory effects in certain animal experiments and the use of these molecules for treatment of autoimmune diseases could become a safer helminthbased method of treatment [5].

\section{CONCLUSION}

Although helminths have been around for thousands of years, humans are just beginning to understand where they and other "unsanitary" organisms may actually help people to stay healthy in certain ways. The hygiene hypothesis and the old friends hypothesis have led to research into the connections between autoimmune disorders and helminth infections. This research is beginning to shift the way that the medical community looks at these worms. So far, research is pointing to the possibility of helminths to regulate the immune system, and through this activity helminths may play a role in the decreasing the symptoms of autoimmune disorders. Helminth products, in the form of certain molecules, may also have beneficial effects in inflammatory diseases and may be safer for patients than giving them living worms.

\section{REFERENCES}

1. Rook, G. A. W., and Brunet, L. R. 2005. Old friends for breakfast. Clin. Exp. Allergy 35:841-2. doi:10.1111/j.1365-2222.2005.02112.x

2. Stiemsma, L. T., Reynolds, L. A., Turvey, S. E., and Brett Finlay, B. 2015. The hygiene hypothesis: current perspectives and future therapies. Immunotargets Ther. 4:143-57. doi:10.2147/ITT.S61528

3. Varyani, F., Fleming, J. O., and Maizels, R. M. 2017. Helminths in the gastrointestinal tract as modulators of immunity and pathology. Am. J. Physiol. Gastrointest. Liver Physiol. 312:G537-49. doi:10.1152/ajpgi.00024.2017

4. Velasquez-Manoff, M. 2016. The Parasite Underground. N Y Times Mag. p. 1-10. Available at: https://www.nytimes.com/2016/06/19/magazine/the-parasiteunderground.html?_r=0 (Accessed: August 27, 2017)

5. Mbanefo, E. C., Le, L., Pennington, L. F., Odegaard, J. I., Jardetzky, T. S., Alouffi, A., et al. 2018. Therapeutic exploitation of IPSE, a urogenital parasite-derived host modulatory protein, for chemotherapy-induced hemorrhagic cystitis. FASEB J. fj.201701415R. doi:10.1096/fj.201701415R 
SUBMITTED: 9 March 2018; ACCEPTED: 14 June 2018;

PUBLISHED ONLINE: 02 July 2018.

EDITED BY: Tansy C Hammarton, University of Glasgow, United Kingdom

CITATION: Tunnessen NOZ and Hsieh MH (2018) Eating Worms to Treat Autoimmune Diseases? Front. Young Minds 6:32. doi:10.3389/frym.2018.00032

CONFLICT OF INTEREST STATEMENT: The authors declare that the research was conducted in the absence of any commercial or financial relationships that could be construed as a potential conflict of interest.

COPYRIGHT (C) 2018 Tunnessen and Hsieh. This is an open-access article distributed under the terms of the Creative Commons Attribution License (CC BY). The use, distribution or reproduction in other forums is permitted, provided the original author(s) and the copyright owner are credited and that the original publication in this journal is cited, in accordance with accepted academic practice. No use, distribution or reproduction is permitted which does not comply with these terms.

\section{REVIEWED BY}

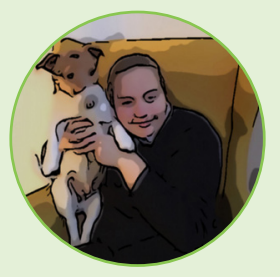

ANNIE, AGE: 14

I am interested in current affairs and I love to read novels. I like music and spending time with my friends. My favorite subjects at school are biology and geography. I love my two cats and my dog.

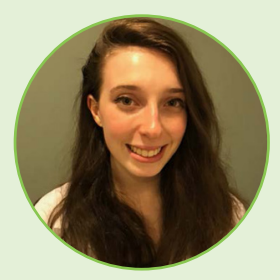

\section{AUTHORS}

\section{NERISSA ORA ZYSKIND TUNNESSEN}

Nerissa Ora Zyskind Tunnessen is a high school senior at the School Without Walls High School in Washington, DC. She plans to attend Vassar College beginning in the fall of 2018. Nerissa is interested in the interactions between helminths and the immune system and she has been studying the hygiene hypothesis. Nerissa has been working with the Bladder Immunology Group Lab at the Biomedical Research Institute in Rockville, MD.

\section{MICHAEL H. HSIEH}

Michael H. Hsieh is the Stirewalt Scientific Director of the Biomedical Research Institute and an Associate Professor at the George Washington University, where he studies host-pathogen interactions in the urinary tract. Michael has published over 90 peer-reviewed scientific papers. His work has been featured on PBS and in the New York Times. *mhsieh@afbr-bri.org 